\title{
Movement velocity vs. strength training
}

\author{
Mário C. Marques*
}

EDITORIAL

Exercise intensity during strength training has been commonly identified with relative load (percentage of onerepetition maximum, 1RM) or with the maximal load that can be lifted a given number of repetitions in each set (e. g., 5RM, 10RM, 15RM). However, these methods appear to have some potential disadvantages (González-Badillo \& Sánchez-Medina, 2010) and, consequently, none of them seem to be appropriate for precisely monitoring the real training effort during resistance exercises in each training session (González-Badillo, 1991).

The first approach requires coaches to individually assess the 1RM value for each athlete. It is true that expressing training intensity as a percentage of the 1RM has the advantage that it could be used to program the strength training for multiple athletes simultaneously, the loads being later transformed in absolute values $(\mathrm{kg})$ for each individual. Further, another important advantage is that this way of expressing training intensity (\% of 1RM) can clearly reflect the evolution of the training load during a training period, provided that we understand the percentage of 1RM as an initial level of effort or degree of demand, and not as a simple arithmetic calculus. Nevertheless, direct assessment of 1RM has some possible disadvantages worth noting. It may be associated with risk of injury when perform incorrectly or by novice athlete's and it is time-consuming and impractical for large groups. Moreover, the actual RM can change quite rapidly after only a few training sessions and often the obtained value is not the subject's true maximum (González-Badillo \& SánchezMedina, 2010).

Another classic way to prescribe loading intensity is to determine, through trial and error, the maximum number of repetitions that can be performed with a given submaximal weight. For example, 5RM refers to a weight that can only be lifted five times, but no more. Some studies identified the relationship between selected percentages of $1 \mathrm{RM}$ and the number of repetitions to failure, establishing a repetition maximum continuum. It is believed that certain performance characteristics are best trained using specific RM load ranges. This method eliminates the need for a direct 1RM test, but it is not without drawbacks either. Using exhaustive efforts is common practice in strength training, but increasing evidence (Davies, Orr, Halaki, \& Hackett, 2016; Izquierdo et al., 2006; ParejaBlanco et al., 2016; Sampson \& Groeller, 2016; Sanborn et al., 2000) shows that training to repetition failure does not necessarily produce better strength gains and that may even be counterproductive by inducing excessive fatigue, mechanical, metabolic and hormonal stress (GonzálezBadillo et al., 2016; Pareja-Blanco et al., 2016; SánchezMedina \& González-Badillo, 2011). In fact, fatigue associated with training to failure not only significantly reduces the force that a muscle can generate, but also the nervous system's ability to voluntarily activate the muscles (Häkkinen, 1993). Consequently, this approach, besides being very tiring and having shown no advantage over other training configurations with lower levels of effort, is unrealistic because it is practically impossible to know exactly how many repetitions can be done with a given absolute load without any initial reference. In addition, if in the first set the subject has completed the maximum number of possible repetitions, it will be very difficult or even impossible to perform properly the same number of reps in the following sets (MacDougall et al., 1999; Richmond \& Godard, 2004).

Movement velocity is another variable which could be of great interest for monitoring exercise intensity during strength training, but surprisingly it has been vaguely mentioned in most studies to date. The importance of monitoring movement velocity for strength training programming was already mentioned in 1991 by professor González-Badillo (González-Badillo, 1991) when valid and reliable instruments for movement velocity measurement were not yet available. In that year, the professor GonzálezBadillo wrote the following: "If we could measure movement velocity each day in real time, this could possibly be the best reference to know if the weight is suitable or not. In addition, we could have recorded the movement velocity for each percentage of 1RM in each subject and, based on this, assess the effort: a movement velocity much lower than the one obtained in other training sessions with the same percentage of $1 R M$ would indicate that the subject is training above the predicted effort; conversely, if movement velocity is higher, it is possible that the subject is above its best performance and, therefore, the training effort performed is less than scheduled".

More recently, González-Badillo and one of his brilliant PhD students Sánchez-Medina (González-Badillo \& Sánchez-Medina, 2010; Sánchez-Medina \& GonzálezBadillo, 2011) studied this hypothesis and confirmed that movement velocity provides as a determinant of the level of effort during resistance training as well as an indicator of the degree of fatigue. Unfortunately, the lack of use of this variable is likely because until recently it was not possible to accurately measure velocity in isoinertial strength training exercises/movements. Indeed, most research that

* Corresponding author: Departamento de Ciências do Desporto da UBI, Rua Marquês Ávila e Bolama, 6201001, Covilhã, Portugal.E-mail: mmarques@ubi.pt 
has addressed movement velocity in strength training have been conducted using isokinetic apparatus which, unfortunately, is not an ideal or common training practice. The actual velocity performed in each repetition could be the best reference to determine accurately the real mechanical and metabolic effort for each athlete. The higher the voluntary velocity achieved against a given (absolute) load, the greater the training intensity (level of effort), which could have positive consequences for training effect (González-Badillo \& Ribas, 2002). Therefore, movement velocity should be the main "ingredient" for determining and monitoring training intensity. In addition, a recent study (González-Badillo, Yañez-García, MoraCustodio, \& Rodríguez-Rosell, 2017) has shown that, rather than prescribing a fixed number of repetitions to perform with a given load, training volume during strength training should be monitored using the magnitude of velocity loss attained in each exercise set because it is closely linked to the actual level of effort being incurred. With this approach, instead of a certain amount of weight to be lifted, coaches must be encouraging to prescribe strength training according to two important variables: 1) first repetition's mean velocity, which is intrinsically related to loading intensity (González-Badillo \& SánchezMedina, 2010); and 2) a maximum percent velocity loss to be allowed in each set (González-Badillo et al., 2017). When this percent loss limit is exceeding the set must be terminated. The limit of repetition velocity loss should be set beforehand depending on the primary training goal being pursued, the particular exercise to be performed as well as the training experience and performance level of each athlete (González-Badillo et al., 2017; SánchezMedina \& González-Badillo, 2011).

\section{REFERENCES}

Davies, T., Orr, R., Halaki, M., \& Hackett, D. (2016). Effect of Training Leading to Repetition Failure on Muscular Strength: A Systematic Review and Meta-Analysis. Sports Medicine (Auckland, N.Z.), 46(4), 487-502. https://doi.org/10.1007/s40279-015-0451-3

González-Badillo, J. J. (1991). Halterofilia. Madrid: Comité Olímpico Español.

González-Badillo, J. J., \& Ribas, J. (2002). Bases de la programación del entrenamiento de fuerza. Barcelona: INDE publicaciones.

González-Badillo, J. J., Rodríguez-Rosell, D., SánchezMedina, L., Ribas, J., López-López, C., MoraCustodio, R., ... Pareja-Blanco, F. (2016). Short-term Recovery Following Resistance Exercise Leading or not to Failure. International Journal of Sports Medicine,
37(4), 295-304. https://doi.org/10.1055/s-00351564254

González-Badillo, J. J., \& Sánchez-Medina, L. (2010). Movement velocity as a measure of loading intensity in resistance training. International Journal of Sports Medicine, 31(5), 347-352. https://doi.org/10.1055/s0030-1248333

González-Badillo, J. J., Yañez-García, J. M., Mora-Custodio, R., \& Rodríguez-Rosell, D. (2017). Velocity Loss as a Variable for Monitoring Resistance Exercise. International Journal of Sports Medicine, 38(3), 217-225. https://doi.org/10.1055/s-0042-120324

Häkkinen, K. (1993). Neuromuscular fatigue and recovery in male and female athletes during heavy resistance exercise. International Journal of Sports Medicine, 14(2), 53-59. https://doi.org/10.1055/s-2007-1021146

Izquierdo, M., Ibañez, J., González-Badillo, J. J., Häkkinen, K., Ratamess, N. A., Kraemer, W. J., ... Gorostiaga, E. M. (2006). Differential effects of strength training leading to failure versus not to failure on hormonal responses, strength, and muscle power gains. Journal of Applied Physiology (Bethesda, Md.: 1985), 100(5), 1647-1656.

https://doi.org/10.1152/japplphysiol.01400.2005

MacDougall, J. D., Ray, S., Sale, D. G., McCartney, N., Lee, P., \& Garner, S. (1999). Muscle substrate utilization and lactate production. Canadian Journal of Applied Physiology = Revue Canadienne De Physiologie Appliquee, 24(3), 209-215.

Pareja-Blanco, F., Rodríguez-Rosell, D., Sánchez-Medina, L., Ribas-Serna, J., López-López, C., Mora-Custodio, R., ... González-Badillo, J. J. (2016). Acute and delayed response to resistance exercise leading or not leading to muscle failure. Clinical Physiology and Functional Imaging. https://doi.org/10.1111/cpf.12348

Richmond, S. R., \& Godard, M. P. (2004). The effects of varied rest periods between sets to failure using the bench press in recreationally trained men. Journal of Strength and Conditioning Research, 18(4), 846-849. https://doi.org/10.1519/14833.1

Sampson, J. A., \& Groeller, H. (2016). Is repetition failure critical for the development of muscle hypertrophy and strength? Scandinavian Journal of Medicine \& Science in $\quad$ Sports, 26(4), 375-383. https://doi.org/10.1111/sms.12445

Sanborn, K., Boros, R., Hruby, J., Schilling, B. K., O’Bryant, H. S., Johnson, R. L., ... Stone, M. H. (2000). ShortTerm Performance Effects of Weight Training With Multiple Sets Not to Failure vs. a Single Set to Failure in Women. Journal of Strength \& Conditioning Research, 14(3), 328- 331.

Sánchez-Medina, L., \& González-Badillo, J. J. (2011). Velocity loss as an indicator of neuromuscular fatigue during resistance training. Medicine and Science in Sports and Exercise, 43(9), 1725-1734. https://doi.org/10.1249/MSS.0b013e318213f880 